\title{
Dendroécologie du genévrier thurifère (Juniperus thurifera L.) : exemple de la thuriféraie de la montagne de Rié (Pyrénées, France)
}

\author{
Valérie Bertaudière $^{\mathrm{a}^{*}}$, Nicolas Montes $^{\mathrm{a}}$, Thierry Gauquelin ${ }^{\mathrm{a}}$, Jean-Louis Édouard ${ }^{\mathrm{b}}$ \\ ${ }^{a}$ Laboratoire d'écologie terrestre, UMR 5552 (CNRS/UPS), 39, allées Jules Guesde 31062 Toulouse cedex, France \\ ${ }^{\mathrm{b}}$ Institut méditerranéen d'écologie et de paléoécologie, Upressa-CNRS 6116, faculté St-Jérôme, case 451,
} 13397 Marseille cedex 20, France

(Reçu le $1^{\text {er }}$ février 1999 ; accepté le 16 août 1999)

\begin{abstract}
Dendroecology of thuriferous juniper (Juniperus thurifera L.): example from a French Pyrenean site at Rie mountain. According to its distribution area (western Mediterranean basin) and its current and past use, the cultural and biogeographical interest of Juniperus thurifera is today recognised. A dendroecological study was carried out to better understand the radial growth responses to climate and the population dynamics of a stand submitted to rural activity decline. According to the comparison of interannual variations in radial growth with monthly climatic parameters (response functions and pointer-year analysis), the influences on the annual tree-ring width of water availability during the current summer and other climatic conditions during the previous autumn were demonstrated. The occurrence of double rings were correlated with variations in summer precipitation. This may emphasise the capability of thuriferous juniper to react promptly to precipitation events in xerothermic conditions associated with peculiarities of the sub-Mediterranean climate. The width and the interannual structure of tree rings indicated that thuriferous juniper growth is strongly correlated with climate variability. An extended dendrochronological study considering its distribution range would allow us to decipher the species' autecological peculiarities. (C) 1999 Inra/Éditions scientifiques et médicales Elsevier SAS.
\end{abstract}

Juniperus thurifera / dendroecology / double ring / Pyrenees / France

Résumé - Le genévrier thurifère, cupressacée arborescente développée essentiellement en milieu montagnard méditerranéen, revêt, en raison de son aire de répartition (bassin méditerranéen occidental) et de son utilisation actuelle et passée, un intérêt biogéographique et culturel reconnu. L’étude dendroécologique conduite ici sur l'un des peuplements français pyrénéens (montagne de Rié) a pour objectif de mettre en évidence les relations entre la croissance radiale de cet arbre et le climat, afin de mieux comprendre le comportement de ces peuplements, menacés en France par la déprise pastorale et agricole.

La confrontation des variations interannuelles d'épaisseur des cernes avec les paramètres climatiques mensuels (fonctions de réponse et années caractéristiques) permet de souligner l'assujettissement de la croissance radiale annuelle au bilan hydrique estival et aux conditions climatiques de l'automne précédent. La fréquence élevée de cernes doubles, reliée aux variations des précipitations mensuelles estivales, traduirait la capacité de l'arbre à optimiser sa croissance et à s'adapter physiologiquement à cette station xérothermique de climat sub-méditerranéen.

La variabilité de l'épaisseur comme de la structure du cerne apparaît donc fortement corrélée aux fluctuations interannuelles du climat. Cette sensibilité du Genévrier thurifère au climat en fait une espèce propice à l'analyse dendrochronologique ; une étude élargie à la totalité de son aire de répartition permettrait d'accéder à son autécologie. C 1999 Inra/Éditions scientifiques et médicales Elsevier SAS.

Juniperus thurifera / dendroécologie / cerne double / Pyrénées / France

\footnotetext{
* Correspondence and reprints
}

bertaudi@cict.fr 


\section{Introduction}

Les formations ouvertes à Genévrier thurifère constituent des formations arborées originales dans le bassin occidental de la Méditerranée. Présent au Maroc, en Algérie, en Espagne, et plus sporadiquement dans le sud de la France (Alpes du Sud, Pyrénées) [13], ce genévrier est aujourd'hui fortement menacé, aussi bien dans son aire de répartition nord-africaine qu'européenne. Dans les Atlas marocains, la dégradation anthropique est importante et la régénération des peuplements est très faible. $\mathrm{Si}$ les causes réelles de cette régénération insuffisante (forte pression anthropique, changements climatiques) sont mal connues, les conséquences de la régression du genévrier thurifère sont déjà observables dans les zones les plus arides : l'accentuation locale des processus érosifs conduit non seulement à la désertification de ces milieux d'altitude, et, plus en aval, à l'ensablement du sud de l'Atlas, mais également à une augmentation de la précarité de la vie des populations berbères, et à un exode rural inéluctable, issu de l'appauvrissement des ressources en bois [12].

En France, le Genévrier thurifère subit les conséquences de la déprise pastorale et agricole. Il est en effet concurrencé par des essences arborées plus compétitives (pins, chênes) qui recolonisent certaines de ses stations, entraînant sa régression et son cantonnement à des zones refuges escarpées $[14,25]$. Par ailleurs, sa faible étendue sur tout le territoire français, et son originalité d'un point de vue systématique dans les Pyrénées [13], en font une relative « rareté botanique », qu'il convient de préserver des incendies ou de tout autre facteur destructif des peuplements.

Les actions de conservation qui ont été engagées sur les peuplements des Pyrénées et des Alpes françaises témoignent aujourd'hui d'un intérêt patrimonial reconnu, même si, dès 1924 déjà, sous l'impulsion d'Ernest Guinier, la célèbre thuriféraie de St-Crépin, acquise par l'Engref, devenait avant l'heure une véritable réserve naturelle [25]. Les thuriféraies françaises sont, en effet, considérées comme " habitats prioritaires " (code CORINE Biotopes 42) par la « Directive Habitats » de la CEE, et le peuplement de la montagne de Rié (Pyrénées centrales, France) a obtenu récemment le statut de «Réserve biologique forestière ».

La compréhension de la dynamique de ces junipéraies passe nécessairement par une connaissance approfondie de l'écologie de cette espèce encore très peu étudiée. L'évaluation de ses exigences vis-à-vis du climat devrait notamment permettre d'en comprendre la répartition spatiale et de préciser ses potentialités. Or, cette cupressacée n'a fait, jusqu'à maintenant, l'objet d'aucune recherche dendrochronologique. En effet, pour toute l'étendue de

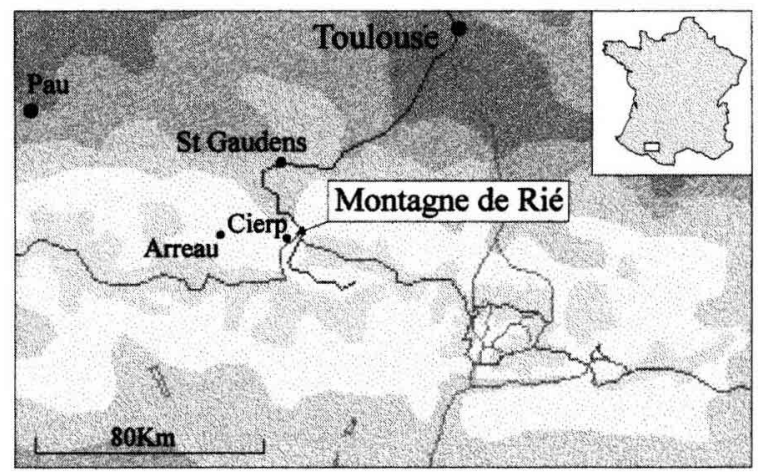

Figure 1. Carte de localisation du site d'étude (Thuriféraie de la montagne de Rié, Pyrénées, France).

son aire de répartition, aussi bien en Afrique du Nord qu'en Europe, l'analyse bibliographique révèle un manque général de données sur la croissance de l'espèce et sur sa sensibilité au climat. Aussi, il s'avérait intéressant d'entreprendre une étude dendroécologique pour mieux appréhender la longévité de cette essence, l'âge des peuplements, et identifier les paramètres climatiques intervenant sur la croissance radiale.

Des études dendroécologiques ont donc été engagées, d'une part sur des thuriféraies marocaines, d'autre part sur un peuplement français pyrénéen. Les travaux exposés ici, focalisés sur la seule thuriféraie de la montagne de Rié (Haute-Garonne, France), présentent les premiers résultats obtenus.

\section{Matériel et méthodes}

\subsection{Site d'étude}

La thuriféraie de la montagne de Rié, d'une superficie de trois hectares, est l'une des deux seules populations importantes de cette espèce connues à ce jour dans les Pyrénées, la deuxième localité étant celle du Quié de Lujat, récemment découverte par Guerby [16]. Relativement dense et peu dégradée, elle s'étend de $650 \mathrm{~m}$ à $1000 \mathrm{~m}$ d'altitude, sur des pentes abruptes (supérieures à $30^{\circ}$ ), selon une bande étroite longeant la crête orientée est-ouest. Elle se présente sous forme d'un piqueté d'arbres ou d'arbustes de petite taille, principalement de forme conique. Les houppiers sont très denses et les troncs, de faible diamètre, très branchus. À l'est et dans la partie culminale, elle est bordée par une chênaie pubescente, qui vient se mélanger au genévrier thurifère 
dans des secteurs où la topographie le permet. Les dynamiques respectives de la thuriféraie et de la chênaie sont marquées par des phénomènes de concurrence entre les deux espèces. Aujourd'hui, le thurifère est replié dans des zones refuges au relief très escarpé [14], qui ne présentent pas de sols assez profonds et suffisamment évolués pour le développement de Quercus pubescens, espèce plus exigeante sur le plan édaphique.

Le peuplement actuel est relativement jeune, les individus ayant moins de 50 ans étant très largement majoritaires, et les plus âgés ne dépassant guère 150 ans [4].

Le poste météorologique le plus proche (Cierp, altitude $495 \mathrm{~m}$ ), situé à $2,5 \mathrm{~km}$ de la station, reçoit des précipitations moyennes annuelles de $1050 \mathrm{~mm}$, relativement bien réparties sur l'ensemble de l'année, mais montrant une diminution significative de juin à septembre. Juillet est relevé comme le mois le plus sec et le plus chaud (moyenne des maxima : $25{ }^{\circ} \mathrm{C}$ ). Cependant, le diagramme ombrothermique ne montre pas de mois sec au sens de Gaussen $(P<2 \mathrm{~T})$ [2] (figure 2). En raison de sa position abritée des vents pluvieux d'ouest par le massif de la Barousse et de sa situation à l'arrière du front pyrénéen, les précipitations annuelles dont bénéficie cette station à Genévrier thurifère seraient cependant plus faibles, comprises entre $700 \mathrm{~mm}$ et $800 \mathrm{~mm}$. La station est soumise, d'autre part, par son exposition, aux vents chauds du sud qui empruntent le Val d'Aran [7].

Si l'été est chaud et sec, l'hiver est relativement rigoureux, avec trois mois présentant une moyenne des minima inférieure à $0^{\circ} \mathrm{C}$ (décembre, janvier, février), le mois de janvier étant le plus froid, avec une moyenne des minima de $-0,7^{\circ} \mathrm{C}$.

Le caractère xérothermique de la station est renforcé par la nature du substrat. La roche-mère, constituée de marbres de calcaire dur, est recouverte d'un sol très superficiel (épaisseur inférieure à $40 \mathrm{~cm}$ ), quand elle n'est pas affleurante. Cette junipéraie est ainsi définie comme un sous-type de station « calcique à calcaire assez chaud et très sec » dans la typologie des stations forestières des Pyrénées centrales proposée par Savoie [35].

\subsection{Méthodes}

\subsubsection{Acquisition des données}

À l'aide de tarières de Pressler, vingt trois arbres ont été échantillonnés, à raison de deux carottes par arbre, orientées à $180^{\circ}$ l'une de l'autre, parallèlement à la courbe de niveau. Les prélèvements ont été effectués vers la base, à des hauteurs variables selon la configuration du terrain et les particularités individuelles de chaque tronc (blessures, ramifications). Les carottes extraites ont été

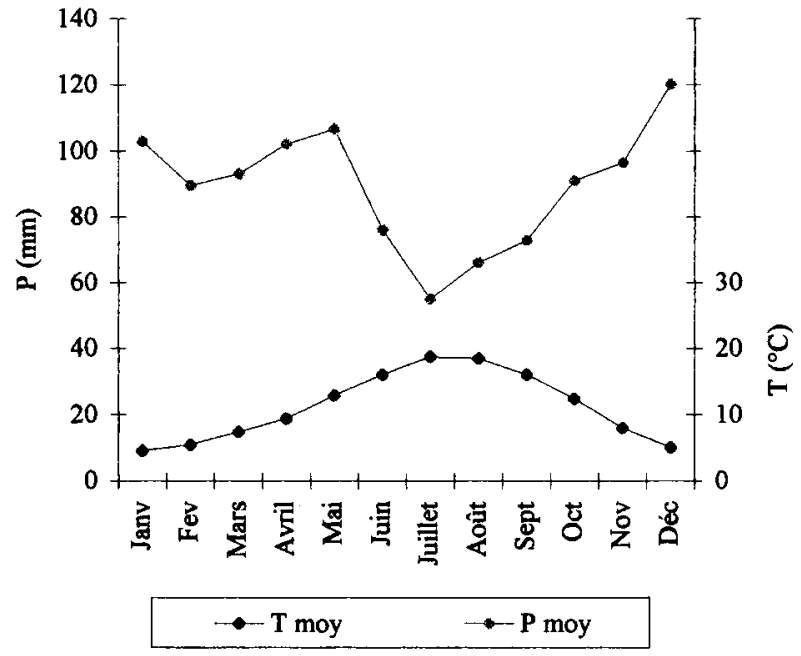

Figure 2. Diagramme ombrothermique du poste météorologique de Cierp (Haute-Garonne, France). (Moyenne : 19601989).

préparées selon les techniques classiques [41]. Quatre arbres morts sur pied, sectionnés à $40 \mathrm{~cm}$ au-dessus du sol, ont fourni également quatre sections transversales de tiges sans préjudice pour le peuplement.

L'interdatation des échantillons a été effectuée directement sur les carottes surfacées selon une coupe radiale [47]. En raison de la fréquence élevée de perturbations visibles de la croissance : cernes discontinus, faux cernes et fluctuations intra-annuelles de densité [23], il s'est avéré très utile de prendre en compte, non seulement les variations relatives d'épaisseur des cernes, mais aussi des caractères tels que la couleur et l'épaisseur relative du bois final. Cette interdatation minutieuse a, de plus, été contrôlée avec succès sur des carottes de pin sylvestre prélevées dans une petite plantation en contrebas de la thuriféraie. Un ultime contrôle, de type graphique [36] et statistique (programme Cofecha [22]), a été effectué sur les séries élémentaires d'épaisseurs des cernes après leur mesure $(1 / 100 \mathrm{~mm})$.

Finalement, seize individus ont été retenus pour la suite de l'étude, leurs séries élémentaires parfaitement datées couvrant au total la période 1839-1996 (figure 3).

\subsubsection{Traitements des séries dendrochronologiques}

L'ensemble des informations apportées par les 32 séries chronologiques retenues est tout d'abord sommairement synthétisée par quelques paramètres statistiques 
simples dont on trouvera la définition détaillée dans Kaennel et al. [23] :

- l'accroissement annuel moyen permet d'évaluer la vitalité du peuplement ;

- la sensibilité moyenne fournit une évaluation chiffrée de la variabilité de l'accroissement radial d'une année à la suivante ;

- le coefficient d'interdatation, directement dérivé du coefficient précédent [30] fournit une évaluation chiffrée du synchronisme des variations interannuelles ;

- le coefficient d'autocorrélation d'ordre 1 est une évaluation des liens qui peuvent exister entre deux cernes successifs.

\subsection{Relations cerne - climat}

L'identification des relations cerne - climat s'appuie sur deux approches : l'une analytique, qui consiste à identifier les années de croissance les plus exceptionnelles et à en rechercher le déterminisme climatique, l'autre de type statistique, qui permet d'établir à travers la fonction de réponse le comportement moyen de l'essence vis-à-vis du climat.

La première approche s'appuie sur le concept d'année caractéristique. Une année est définie comme " caractéristique » au sens large, lorsque l'occurrence d'un cerne diagnostique donné (ils ont été définis dans la phase d'interdatation) se répète sur l'ensemble des chronologies [23]. Outre les caractères qualitatifs (faux-cerne, cerne absent), le cerne diagnostique peut être identifié par une épaisseur exceptionnelle (positive ou négative) ; L'année caractéristique marque donc, sur la station ou l'aire considérée, l'intervention très contraignante d'un facteur, qui est presque toujours associé au climat [38].

Une définition plus restrictive de l'année caractéristique repose sur des données quantitatives. Une année est dite caractéristique lorsque, pour deux années successives, on constate une augmentation ou une diminution concordantes de l'épaisseur du cerne, affectant un certain pourcentage de chronologies. Le pourcentage à retenir dépend de la taille de l'échantillon selon l'équation de Graf et Henning (in [9]), c'est-à-dire du nombre de séries élémentaires représentant chaque année. Le seuil retenu ici est $80 \%$, valeur seuil significative à $99 \%$. Le qualificatif d'année caractéristique extrême a été affecté aux années pour lesquelles l'épaisseur du cerne est supérieure ou inférieure à $m+\sigma$ ou $m-\sigma$, m étant l'accroissement moyen calculé sur toute la population et $\sigma$ l'écart-type correspondant [33] (figure 4). Sont aussi définis des maxima et minima caractéristiques, afin de pallier le phénomène de résonance : un cerne d'épaisseur moyenne

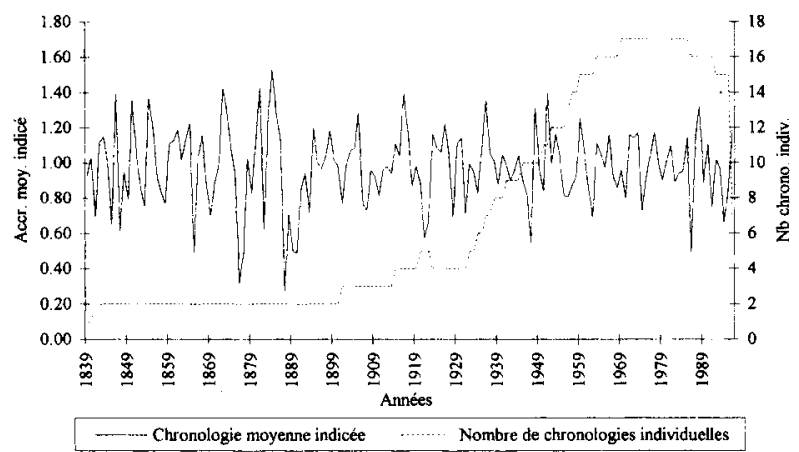

Figure 3. Chronologie maîtresse indicée de la thuriféraie de la montagne de Rié (Pyrénées, France).

peut, en effet, apparaître très large s'il est précédé d'un cerne très étroit et inversement. Selon Munaut [30], lorsqu'une année de tendance négative succède à une année positive, cela définit un maximum caractéristique et, dans le cas inverse, un minimum caractéristique.

Les années caractéristiques ainsi définies sont alors confrontées aux données climatiques des années considérées, afin de mettre en évidence les relations entre l'épaisseur du cerne et l'influence d'un paramètre climatique extrême.

La figure 4 permet également de repérer les années caractéristiques à cerne double. Les cernes doubles, ou fluctuations intra-annuelles de densité [23], observées en grand nombre chez le Genévrier thurifère, se caractérisent, dans le cas présent, par la formation d'une bande de cellules de petit diamètre à paroi épaisse au cours de l'élaboration du bois final ou plus rarement du bois initial. De la comparaison des deux carottes d'un même individu et des différents arbres entre eux, il ressort que, dans certains cas, ces fluctuations de densité sont très accentuées et leurs limites nettement marquées. Elles définissent alors, pour une même année, deux cernes morphologiquement similaires, appelés «faux cernes » [23]. Dans la mesure où, pour une année donnée, cette configuration se répète sur un nombre significatif d'arbres, elle peut être liée à des conditions climatiques particulières $[23,28,32$, 48 ] et considérée comme année caractéristique. Ainsi, plus précisément, une année a été définie comme « année caractéristique », à partir du moment où au moins $20 \%$ des arbres échantillonnés ont présenté indifféremment un cerne double ou un faux-cerne. Le seuil de $20 \%$ a été choisi ici dans la mesure où, en dessous de cette valeur de $20 \%$, sur la période 1945-1996 (période couverte par au moins dix chronologies individuelles) trop d'années sont 


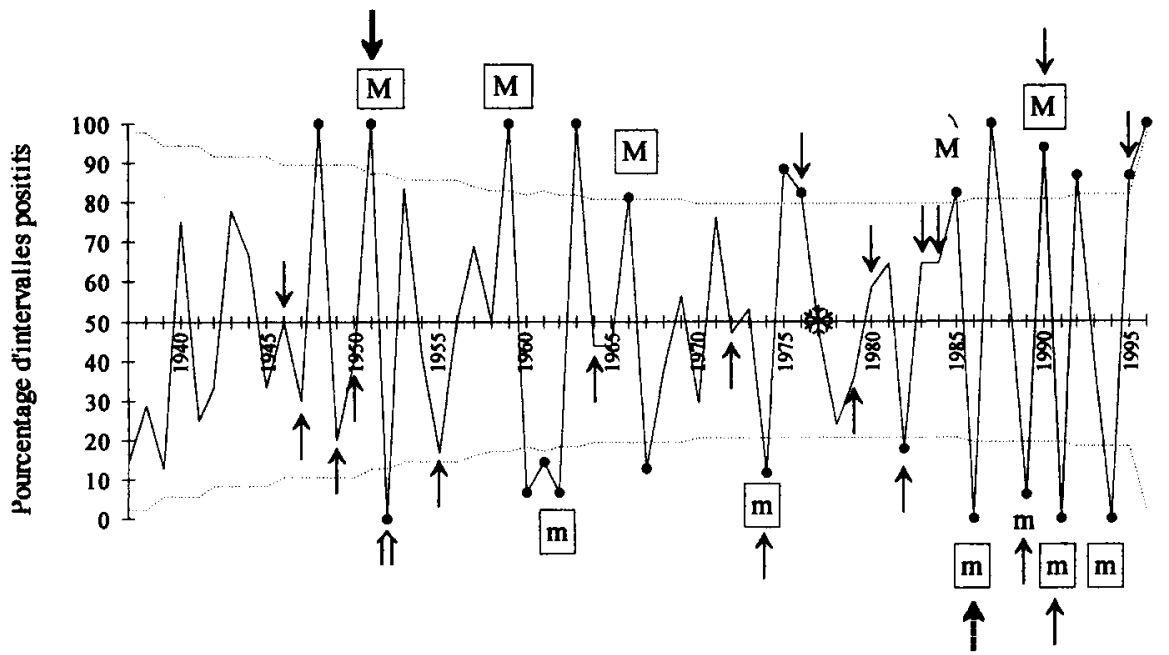

- Annce caractéristique (au seuil minimum $80 \%$ )

m minimum caracteristique

M Maximum caractéristique

Anné caractéristique extrême $(\bar{x}>\bar{x} \pm \sigma)$

Seuil de signification a $99 \%$

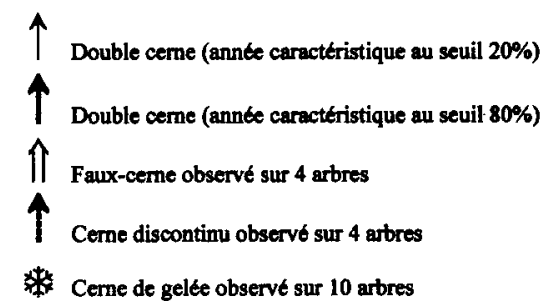

Figure 4. Années caractéristiques définies à partir du pourcentage d'arbres montrant un cerne diagnostique positif ou négatif pour une même année; année à double cerne, à faux-cerne, à cerne discontinu et à cerne de gelée. concernées (figure 5) et ne peuvent alors être considérées, de ce point de vue, comme caractéristiques.

Afin d'identifier plus précisément dans le cas du Thurifère de la montagne de Rié, quels étaient les facteurs climatiques potentiellement responsables de la formation de ces cernes doubles, les précipitations mensuelles et températures moyennes mensuelles des années caractéristiques ont été comparées à celles des années non caractéristiques sur la période 1962-1996 (données MétéoFrance disponibles), comme cela a été fait pour le chêne vert [49].

La seconde approche fait appel au concept de «Fonction de réponse $»[10,17,39]$. Elle est décomposée en deux étapes.

Dans un premier temps, il est en effet nécessaire d'isoler au préalable, dans les séries de cernes, le signal climatique exprimé par les variations interannuelles de haute fréquence. Pour cela, il convient d'éliminer les autres composantes de la variation du cerne, exprimées par des signaux de basse et moyenne fréquence, telles que l'effet à long terme de l'âge de l'arbre et l'ensemble des bruits d'origine variée résultant des caractéristiques indivi- duelles des arbres, de l'évolution des conditions microstationnelles et des phénomènes de compétition [42].

Les séries d'épaisseurs brutes des cernes sont donc transformées en séries d'indices, par filtrage (établissement d'une courbe lissée) puis standardisation (rapport de l'épaisseur mesurée à la valeur correspondante de la courbe lissée théorique ajustée à la série brute) [19].

Les séries élémentaires indicées ont été moyennées afin d'obtenir, pour chaque individu une série individuelle indicée, et, pour la population totale, une série moyenne indicée appelée «chronologie maîtresse » (figure 3).

Dans un second temps, la série moyenne indicée est confrontée à des séries synchrones de paramètres climatiques mensuels représentatifs du climat (précipitations mensuelles combinées aux températures moyennes mensuelles maximales et aux températures moyennes mensuelles minimales) sur une période de douze mois s'étendant du mois d'octobre de l'année précédant la formation du cerne au mois de septembre de l'année de son élaboration, période de construction du cerne généralement admise pour les espèces méditerranéennes $[38,16,42,47$, $30,26]$. 


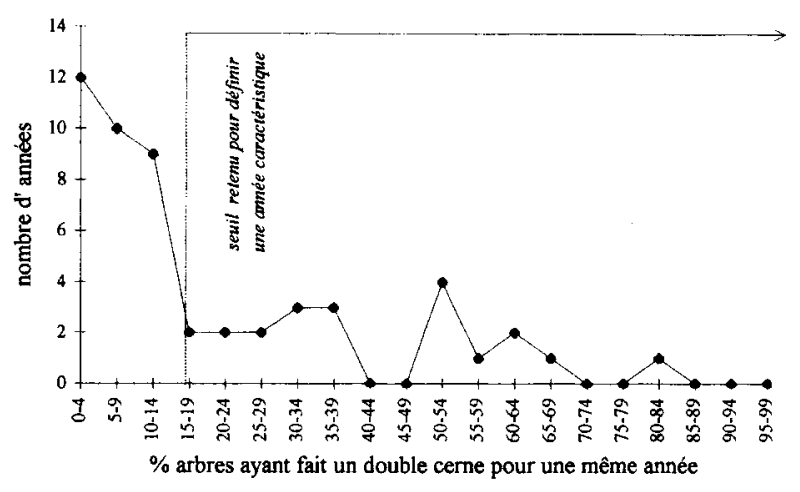

Figure 5. Nombre d'années concernées par chaque classe de pourcentages d'arbres, ayant fait un double cerne pour une même année, sur la période 1945-1995.

Les fonctions de réponse ont été calculées avec le programme Calrob du logiciel PPPHALOS, Programs in Paleoclimatology: Prevision of Hiatus and Analysis of Linkages between Observation and between Series [19]. Le calcul fait appel à une régression orthogonalisée entre la variable dépendante (épaisseur du cerne moyenne indicée) et les variables explicatives (données climatiques mensuelles), résultantes d'une analyse en composante principale des paramètres climatiques. Les coefficients de régression partiels ( $r$ ) obtenus sont ensuite appliqués à la série climatique pour reconstruire la variable dépendante. La valeur prédictive du modèle peut être estimée par le coefficient de corrélation $(R)$ entre les variables dépendantes estimées et les variables réellement observées (épaisseurs des cernes mesurées).

Pour évaluer la fiabilité statistique des fonctions de réponse, le calcul met en œuvre une procédure Boot-strap (tirage au sort avec remise) [18] permettant de répéter le calcul de la régression sur un grand nombre d'échantillons simulés à partir des données initiales. Les calculs de régression sont ainsi effectués sur des années tirées au sort (années de calibration), et sur les années de vérification non sélectionnées lors du tirage. La procédure est répétée cinquante fois et génère ainsi cinquante fonctions de réponse. Cette méthode permet de s'affranchir du postulat de distribution normale associé à l'utilisation des tests d'hypothèse.

Le rapport du coefficient de corrélation moyen $(R)$ à son écart-type $(s)$, pour les deux périodes (calibration et vérification), donne une estimation de la signification statistique globale de la fonction de réponse et exprime la fiabilité de la relation établie entre les variables dépendantes et les variables explicatives. Le signe des coeffi- cients de régressions partiels et le rapport $r / s$ correspondant traduisent respectivement le sens de la relation entre le paramètre climatique et l'épaisseur du cerne, et sa signification statistique. Un signe positif indique une relation directe (l'élaboration d'un cerne large pour des valeurs de la variable climatique supérieures à la moyenne), tandis qu'un signe négatif traduit une relation inverse (la formation d'un cerne étroit pour des valeurs de la variable climatique supérieures à la moyenne).

Compte tenu du faible nombre d'observations, les régresseurs climatiques ont été regroupés afin de rendre la relation «cerne-paramètre climatique » plus stable et plus significative. Les regroupements sont basés dans un premier temps sur des critères biologiques en relation avec la phénologie et les différentes phases de croissance de l'arbre, et dans un second temps, en relation, avec le signe des différents coefficients de régression partiels obtenus $[19,26,48]$.

La période retenue pour les calculs s'étend de 1953 à 1996 ; après avoir contrôlé l'homogénéité climatique de la station de Cierp avec celle d'Arreau, poste météorologique voisin, les données climatiques manquantes de Cierp ont, au préalable, été estimées mathématiquement à partir de cette deuxième station.

\section{Résultats}

\subsection{Caractéristiques dendrochronologiques}

Les caractéristiques dendrochronologiques du peuplement sont synthétisées dans le tableau I.

La croissance radiale du Genévrier thurifère est lente, avec un accroissement moyen de $0,99 \mathrm{~mm} / \mathrm{an}$. Ces genévriers présentent, par ailleurs, en général un cœur très excentré, résultant d'une croissance déséquilibrée sur le pourtour du tronc, et un grand nombre " d'anomalies de croissance » telles que des cernes doubles et des cernes discontinus, des cicatrices laissées par le gel ou le passage du feu.

La moyenne des sensibilités moyennes individuelles de la thuriféraie est relativement élevée $(0,32)$, comparée aux valeurs obtenues pour d'autres espèces européennes présentes dans le bassin méditerranéen (Pinus pinea, Pinus pinaster, Pinus halepensis, Pinus sylvestris, Quercus pubescens), qui varient autour de $0,2[38,44$, 16].

Nous observons ici un coefficient d'interdatation de 0,78 , traduisant une bonne homogénéité des variations interannuelles de la croissance radiale des arbres du peuplement. Ce coefficient est, en effet, d'autant plus élevé que les arbres du peuplement présentent des variations interannuelles synchrones bien marquées. 
Tableau I. Caractéristiques dendrochronologiques de la thuriféraie de la montagne de Rié (Pyrénées, France).
Nombre total d'arbres échantillonnés Nombre d'arbres retenus après interdatation Chronologie totale

Accroissement radial annuel moyen $(\mathrm{mm})$

Moy. des sensibilités moyennes individuelles

Coefficient d'interdatation

Cocfficient d'autocorrélation moyen (ordre 1 )
27

16

$1839-1996$

$0.99(+1-0.29)$

$0.32(+/-0.05)$

0.78

$0.46(+/-0.18)$
Quant au coefficient d'autocorrélation d'ordre 1, il atteint à Rié une valeur moyenne assez élevée de 0,46.

\subsection{Années caractéristiques}

Sur la période 1937-1996 (soit 60 années), le Thurifère présente onze années caractéristiques, définies à partir des maxima et minima caractéristiques, soit une fréquence d'environ $18 \%$ (figure 4). Elles se répartissent en six années à croissance faible (cernes étroits) et cinq années à croissance forte (cernes larges). Parmi elles, neuf années extrêmes ont été dénombrées, dont cinq figurées par un cerne très étroit $(1962,1974,1986,1991$, $1994)$ et quatre par un cerne très large $(1951,1959,1966$, 1990). 1986 est l'année caractéristique la plus prononcée, puisqu'elle correspond non seulement à un cerne très étroit dans $100 \%$ des cas, mais également à un cerne discontinu chez quatre individus.

Il ressort de la confrontation des années caractéristiques extrêmes avec les données météorologiques disponibles, que l'ensemble des années extrêmes négatives est marqué par une période estivale (juin, juillet et août) très sèche et chaude. Les années 1974, 1991 et 1994 présentent, de plus, un printemps (avril, mai) froid.

Les années caractéristiques positives, exprimées par un cerne large, montrent toutes un mois de septembre sec. Parmi elles, les années biologiques 1985 et 1990 sont précédées d'un automne plus chaud que la moyenne.

D'un point de vue qualitatif, l'année caractéristique 1977 se distingue par un cerne de gelée tardive, remarqué sur dix arbres de notre échantillonnage. Aucune année caractéristique n'a été définie à partir de cernes absents, ni de cernes discontinus. En effet, aucun cerne absent n'a été observé, et, quant aux cernes discontinus, ils sont ici peu nombreux, localisés seulement en $1986 \mathrm{chez}$ quatre individus et en 1987 sur un seul échantillon.

En revanche, les années caractéristiques avec fluctuations intraannuelles de densité sont fréquentes. Au seuil retenu $(20 \%)$, on en dénombre au total 19 sur la période
1945-1996, soit $36 \%$. Si l'on considère le seuil de $80 \%$, seule l'année 1951 est concernée, avec neuf individus sur onze montrant un cerne double cette année-là. Les fauxcernes, observés pour les années 1948, 1950, 1952, 1955 , 1971, 1972, 1975, 1980, 1982, 1984, 1995, se répartissent sur un nombre réduit d'arbres (huit individus). Au mieux, quatre arbres présentent un faux-cerne pour une même année (1952).

\subsection{Cerne double et climat}

Le pourcentage d'arbres échantillonnés, ayant fait un cerne double pour une même année, varie de $6 \%$ à $80 \%$. Ces cernes doubles sont visibles dans la majorité des cas sur les deux carottes opposées d'un même individu et peuvent ainsi constituer des cernes diagnostiques, utiles pour l'interdatation. Leur continuité sur tout le pourtour du tronc indique, de plus, que l'arbre semble réagir dans son intégralité aux facteurs induisant leur élaboration.

Sur un ensemble de quinze arbres, l'épaisseur moyenne d'un cerne double est, pour la majorité des individus $(80 \%)$, supérieure à l'épaisseur moyenne du cerne calculée sur les années sans cerne double (figure 6). L'accroissement moyen individuel tend par ailleurs à être d'autant plus fort que le pourcentage de cerne double par individu, variant de 8 à $45 \%$, est élevé. Il atteint toutefois une valeur maximum, pour les arbres dont le pourcentage de cerne doubles est compris entre 20 et $30 \%$. Il est intéressant de noter également que, pour de forts pourcentages de cernes doubles par arbre, l'écart entre l'épaisseur moyenne des cernes doubles et l'épaisseur moyenne individuelle est plus faible. Autrement dit, les cernes doubles apparaissent plus épais, lorsqu'ils sont moins fréquents.

Au-delà du seuil de $30 \%$, les valeurs d'accroissement radial annuel brut diminuent avec l'augmentation du pourcentage de cerne double par arbre (figure 6), mais restent tout de même supérieures à celles des individus à faible taux de cernes doubles. La croissance normale des quatre arbres concernés paraît perturbée négativement.

De la comparaison des paramètres climatiques des années avec ou sans cerne double, il ressort que, bien que les températures moyennes mensuelles des années avec cerne double soient légèrement supérieures à celles des années sans cerne double, elles ne différent pas significativement de ces dernières (test $t$ de Student, seuil $5 \%$ ), et ne semblent donc pas intervenir au niveau de la formation d'un cerne double. Les précipitations mensuelles estivales (juin et août) différent, quant à elles, significativement (95\% de confiance, test $t$ de Student) (figure 7). La succession d'un déficit hydrique en début d'été (juin sec ou juin et juillet cumulés secs) et d'une fin de saison avec des précipitations plus abondantes que la moyenne (août 


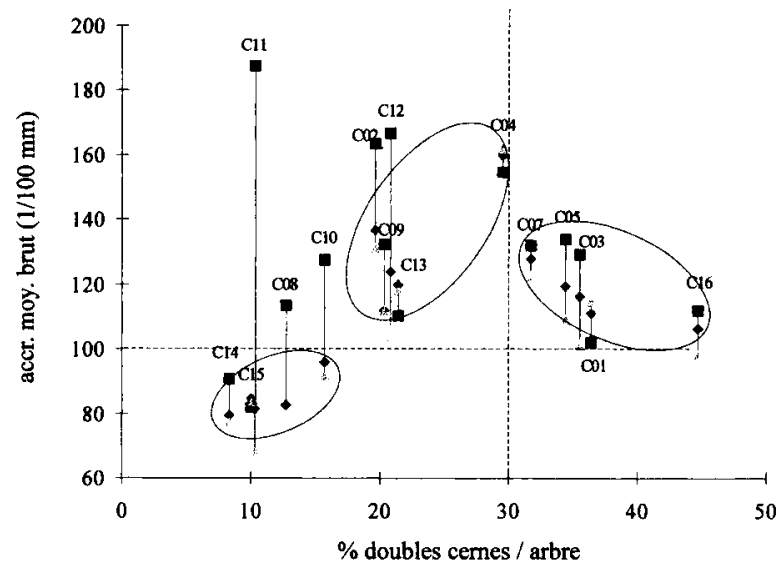

- Epaisseur moyenne de tous les cernes

- Epaisseur moyenne des cernes doubles de la séquence Epaisseur moyenne de tous les cernes moins les cernes doubles

Figure 6. Accroissements radiaux bruts des différents arbres, moyennés sur les années avec ou sans double cerne, en fonction du pourcentage de doubles cernes par individu.

pluvieux ou août et septembre cumulés pluvieux) apparaîtrait être la condition climatique responsable de ces fluctuations intra-annuelles de densité.

\subsection{Fonctions de réponse}

Les fonctions de réponse, qui traduisent les relations « épaisseur du cerne - précipitations et températures maximales (P-Tmax.) » et " épaisseur du cerne - précipitations et températures minimales (P-Tmin.) », sont globalement significatives au seuil $99,9 \%$ pour les périodes de calibration et de vérification. Dans les deux cas, ces relations apparaissent relativement fortes : le coefficient de corrélation multiple calculé sur les années de calibration atteint, en effet, respectivement une valeur de 0,78 (écart-type : 0,04) et 0,71 (écart-type : 0,05), ce même coefficient calculé sur les années de vérification présentant des valeurs respectives de 0,59 (écart-type : 0,14 ) et 0,6 (écart-type : 0,18).

On observe une relation directe forte, significative au seuil $99 \%$, entre l'épaisseur du cerne et les précipitations estivales (juin, juillet, août) de l'année en cours, et une relation inverse avec les températures moyennes maximales et minimales de ces mêmes mois d'été (figure 8 ).

Les conditions hydriques de la fin de la période de croissance ont, elles aussi, une influence importante sur

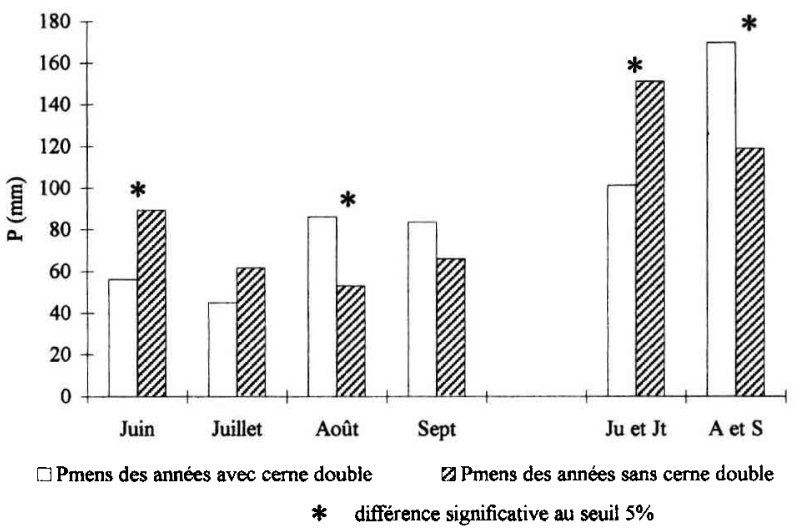

Figure 7. Précipitations mensuelles estivales des années avec ou sans double cerne, moyennées sur la période de 1962-1996 (Station météorologique de Cierp, Haute-Garonne, France).

l'épaisseur du cerne de l'année courante. Le coefficient de régression partiel négatif et significatif au seuil $90 \%$ pour le mois de septembre, observé dans les deux cas ( $\mathrm{P}-$ Tmax et P-Tmin), montre, en effet, que de fortes précipitations au mois de septembre ont une influence négative sur la formation du cerne de l'année.

L'intervention des conditions climatiques hivernales (décembre, janvier, février, mars) apparaît également non négligeable. On observe une relation inverse, significative au seuil $99,9 \%$ entre les précipitations des mois de décembre-janvier et la largeur du cerne à venir, combinée à une influence négative des températures minimales du mois de janvier. De fortes précipitations en février-mars semblent induire, à l'opposé, un cerne large.

Les précipitations printanières (avril, mai), quant à elles, ne montrent aucune relation avec l'épaisseur du cerne.

Seules les températures moyennes maximales et minimales de cette saison paraissent jouer un rôle important dans l'élaboration du cerne. Lorsqu'elles sont élevées, elles induiraient une forte croissance radiale (relations respectivement significatives aux seuils $99,9 \%$ et $99 \%$ ).

Une relation directe, significative au seuil $99,9 \%$, entre l'accroissement radial annuel et les conditions climatiques de l'automne précédant la formation du cerne est également mise en évidence. Des précipitations abondantes en octobre et novembre de l'année précédente, associées à des températures élevées, engendreraient une variation positive de la croissance. 


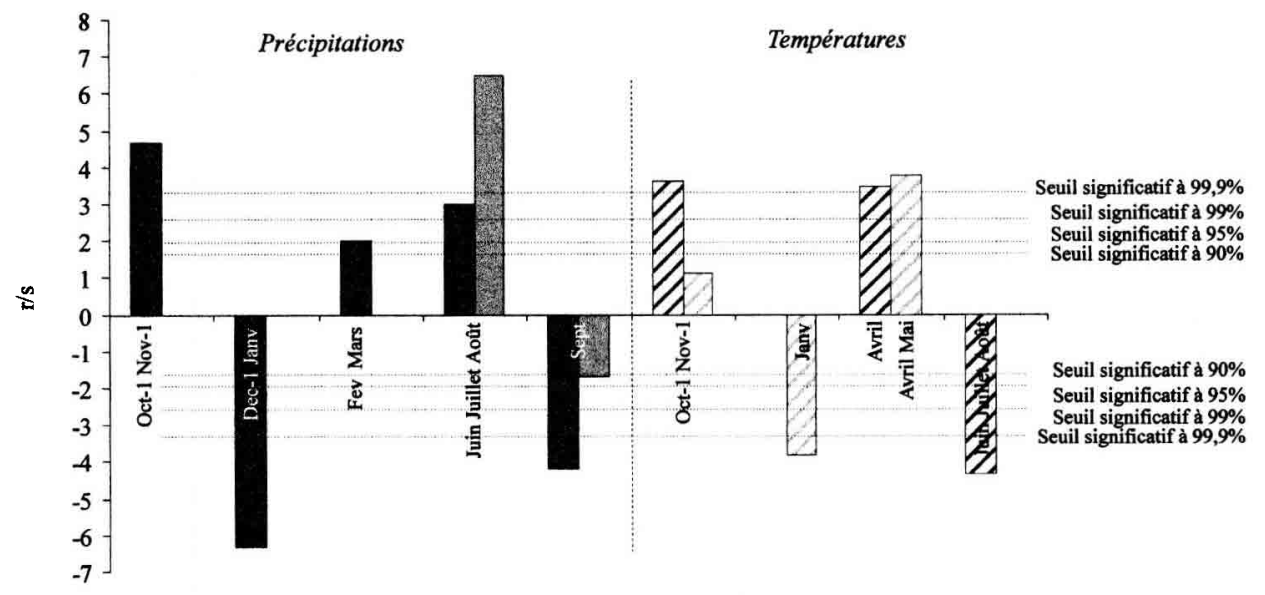

Combinaison (Précipitations $\square+$ Températures maximales $V / \triangle$ )
Combinaison (Précipitations $\square+$ Températures minimales $\square$ )
Figure 8. Fonction de réponse de la thuriféraie de la montagne de Rié (Pyrénées, France) pour les couples P-Tmax et PTmin, significative au seuil $99,9 \%$ (sur les années de calibration et de vérification), pour la période 1953-1996.

\section{Discussion et conclusion}

Le Genévrier thurifère, développé essentiellement dans les milieux montagnards du bassin méditerranéen, se situe à Rié dans la partie occidentale de son aire de répartition. Localisé dans un bassin intramontagnard des Pyrénées centrales [35], les influences océaniques qu'il subit, sont atténuées par le relief, la topographie et les conditions microstationnelles particulières du site (forte pente, substrat calcaire, sol superficiel, exposition sud). L'influence méditerranéenne qui s'exprime au niveau floristique [3] témoigne du caractère fortement xérothermique de cette station.

Cette xéricité du milieu se matérialise, de plus, par la forte influence des conditions hydriques estivales sur la croissance radiale du genévrier. Les fonctions de réponse et l'analyse des années caractéristiques montrent que des précipitations mensuelles estivales faibles, associées à des températures moyennes mensuelles élevées, induisent conjointement des cernes étroits, voire discontinus pour une année de sécheresse très accentuée telle que 1986. La sécheresse climatique et édaphique de l'été, accentuée par une évapotranspiration élevée dans des conditions de fortes chaleurs, semble fortement ralentir la croissance du genévrier. L'activité méristèmatique pourrait même momentanément cesser quand les conditions extérieures deviennent vraiment trop défavorables, comme en témoignent les cernes discontinus observés sur certains individus.

La grande variabilité des épaisseurs des cernes annuels apparaît ainsi significativement liée au bilan hydrique des trois mois d'été (juin, juillet et août).
La présence en grand nombre de cernes doubles sur tous les individus, ayant pu être reliée climatiquement aux variations de ces précipitations mensuelles et plus précisément à la succession d'un début d'été plus sec et d'une fin d'été très arrosée, confirme la relation mise en évidence précédemment. En période estivale, l'arbre semble tirer parti directement de toute pluie d'été pour favoriser sa croissance.

Une telle adaptation physiologique a été mise en évidence chez d'autres espèces du genre Juniperus [21] et chez un autre taxon méditerranéen, Quercus ilex L. [49].

Cependant, le pourcentage élevé de cernes doubles ne s'explique pas toujours par une réponse biologique directe au climat. Chez certains individus à croissance plus faible, le taux de cernes doubles atteignant parfois $45 \%$ (n'ayant pas pu être relié à l'âge) pourrait ici s'expliquer par des conditions microstationnelles très limitantes (arbre poussant sur falaise ou sur dalle rocheuse affleurante peu fissurée, arbre surcîmé par un concurrent et subissant une forte compétition...), qui accentueraient fortement l'action dépressive de la sécheresse édaphique en début d'été et placeraient l'arbre en situation de stress chronique perturbant négativement sa croissance. Dans cette hypothèse, le rôle de l'enracinement de l'arbre et son efficacité deviennent majeurs dans le fonctionnement hydrique de l'individu, comme cela a été souligné dans l'étude du comportement estival du Cèdre de l'Atlas et du Chêne pubescent dans le Mont-Ventoux [1].

Compte tenu du rôle important des facteurs génétiques dans la croissance radiale d'un arbre [28], une relation éventuelle entre le taux de cernes doubles et des facteurs endogènes à l'arbre, qui s'exprimeraient plus fortement 
dans des conditions microstationnelles très limitantes, peut être aussi envisagée.

À Rié, l'activité physiologique du genévrier thurifère est donc réduite pendant les périodes défavorables (alimentation hydrique difficile). L'arbre n'entre cependant pas systématiquement en dormance estivale, contrairement à certaines essences, telles que Pinus sylvestris, Pinus nigra, Pinus pinaster, Pinus uncinata [5], qui évitent la sécheresse en bloquant très tôt leurs activités photosynthétiques.

Un comportement analogue à celui du thurifère a été observé chez d'autres espèces se développant en milieu méditerranéen, telle que Quercus pubescens Willd [43], Quercus ilex L. [49] ou Cedrus atlantica Manetti [1, 5]. Le thurifère semble pouvoir moduler sa croissance en fonction des disponibilités hydriques du milieu et profiter ainsi du climat local. Ce comportement "opportuniste » lui permet, dans le site de Rié, de croître dans des zones pourtant très hostiles au développement d'une espèce arborée (situation de crête rocheuse escarpée), car il sait profiter des précipitations non négligeables de fin d'été, malgré la sécheresse estivale qu'il subit sous ce climat local de type sub-méditerranéen.

Durant la période de formation du cerne (d'avril à septembre-octobre), en dehors des précipitations des mois d'été ou de fin d'été (juin, juillet, août, septembre), la pluviométrie n'intervient apparemment pas de manière significative. On peut penser que, sous ce climat, les précipitations printanières sont assez abondantes pour ne pas être limitantes. De plus, la relation directe entre la croissance de ce genévrier et les précipitations automnales de l'année précédente, combinées à des températures douces durant cette même saison, montre que l'arbre pourrait profiter de ces conditions favorables pour, d'une part, augmenter son système racinaire et, d'autre part, synthétiser et stocker des réserves nutritives utiles au redémarrage de son activité cambiale au printemps suivant. La croissance du tronc et des racines est, en effet, très liée à la quantité de réserves accumulées antérieurement [11].

Cette forte corrélation entre la croissance radiale et le climat antérieur au printemps a été soulignée dans des études dendroécologiques précédentes sur des espèces de milieux arides $[10,32]$ ou de milieu méditerranéen $[16$, 33]. Cependant, la constitution de réserves d'eau dans le sol à l'automne et en hiver ne peut être ici considérée comme un facteur déterminant dans le redémarrage de la croissance, puisque le sol est très superficiel, parfois réduit au comblement d'une faille de la roche-mère dans les situations de falaises, et pauvre en argiles.

La relation inverse entre l'épaisseur du cerne et les précipitations du mois de septembre de l'année de son élaboration ne trouve ici aucune explication immédiate.
Cette corrélation négative, observée également chez le Cèdre de l'Atlas dans le sud-est de la France, a été reliée, dans ce cas là, à la fructification importante certaines années, éventuellement favorisée par les fortes précipitations de septembre et le détournement des substances trophiques qu'elle entraîne, au détriment de la croissance en épaisseur [17]. Le Genévrier thurifère étant un arbre dioïque, cette hypothèse n'est plausible que si cette relation inverse est vérifiée avec des individus femelles uniquement. Or, même si le sexe des arbres étudiés a été systématiquement noté, l'échantillonnage réalisé dans une optique différente et la présence de plusieurs arbres de sexe indéterminé ne nous ont pas permis de la valider.

Parmi les paramètres climatiques mensuels printaniers, seules les températures maximales et minimales du mois d'avril semblent jouer un rôle significatif dans les variations de la croissance radiale du thurifère, et leur influence apparaît synchrone avec la réactivation cambiale printanière. La levée de dormance au printemps, résultant de l'effet direct des températures vernales, associé au photopériodisme [11], ne serait donc pas dépendante des précipitations de cette même saison.

Si des températures moyennes douces en avril semblent favorables à la croissance par une levée de dormance anticipée, un démarrage précoce de l'activité cambiale ne sera pas nécessairement avantageux pour l'arbre, dans le cas où un gel sévère tardif pourrait endommager le cambium en provoquant de fortes déstructurations cellulaires, comme le montrent à Rié des cicatrices laissées par le gel en 1977.

La relation directe négative des températures minimales du mois de janvier avec l'épaisseur du cerne témoigne de la sensibilité de l'arbre aux températures basses extrêmes. Sensible aux froids rigoureux de l'hiver, le Genévrier thurifère montre un comportement hivernal analogue à celui des espèces méditerranéennes dans le sud de la France $[16,43,48]$.

En ce qui concerne la relation inverse entre les précipitations hivernales (décembre-janvier) et l'épaisseur du cerne à venir, l'hypothèse d'un engorgement du sol et de son effet asphyxiant pour les racines ne peut ici être retenue, compte tenu de la nature et de la faible profondeur du sol. Dans l'état actuel des connaissances sur la physiologie de l'espèce, et compte tenu de la difficulté à caractériser le sol sur le plan hydrique dans les conditions étudiées, aucune interprétation valable n'a pu être avancée. Le caractère causal direct des fortes précipitations hivernales ainsi que celles du mois de septembre, associées à croissance radiale amoindrie, est donc peu probable, laissant à penser que d'autres variables corrélées à ces facteurs climatiques, telles que l'ensoleillement par exemple, seraient plus directement impliquées. 
Les relations exclusivement statistiques entre le climat et la croissance radiale doivent par conséquent être interprétées en fonction de paramètres climatiques plus élargis, et, a fortiori, en prenant en compte les caractères édaphiques et structuraux des peuplements. Une modélisation plus «fonctionnelle » de la croissance radiale nécessiterait également des connaissances écophysiologiques approfondies de l'espèce étudiée.

Les conditions climatiques et édaphiques très sévères des milieux colonisés du site de Rié déterminent donc une croissance lente et complexe du Thurifère. Cette faible croissance radiale ne s'observe pas seulement à la montagne de Rié, mais également dans les autres stations françaises des Alpes et de Corse, où les accroissements moyens varient respectivement de $0,9 \mathrm{~mm} / \mathrm{an}$ à $1,6 \mathrm{~mm} / \mathrm{an}$ [25] et de 1,41 à $1,95 \mathrm{~mm} / \mathrm{an}$ [32]. Mais elles imposent aussi aux différents individus de la population, et de manière homogène, une forte variabilité de l'accroissement radial annuel.

Étant donné sa longévité (arbres pluricentenaires au Maroc), le thurifère devient alors une essence très intéressante au point de vue dendrochronologique, comme les sont d'autres espèces du même genre : Juniperus scropulorum, Juniperus virginiana, et Juniperus occidentalis qui se sont ainsi révélées aux États-Unis comme des espèces d'intérêt majeur [15], de même que Juniperus polycarpos en Iran [26], Juniperus phenicea en Israël [46], Juniperus indica au Pakistan [8] ou encore Juniperus excelsa en Oman [10], qui ont permis de constituer des chronologies de plus de trois siècles.

De plus, grâce à l'imputrescibilité du bois de thurifère, les arbres morts sur pied et les souches anciennes se conservent longtemps dans le milieu et permettent, après interdatation, de reconstruire des chronologies plus longues que celles qui sont initialement données par les arbres vivants. Dans la station étudiée, en effet, il a été possible de dater un arbre mort sur pied, resté intact depuis 75 ans.

Espèce montagnarde méditerranéenne à sub-méditerranéenne selon le pays et la région où il s'est développé, le genévrier thurifère, en modulant sa croissance selon les ressources hydriques du milieu, apparaît très adapté aux milieux arides. Par son impressionnante vitalité et sa grande robustesse face aux contraintes environnementales, Juniperus thurifera témoigne de la grande résistance du genre Juniperus aux conditions stationnelles difficiles, déjà soulignée par Fritts [11] à propos de Juniperus virginiana et Juniperus communis. Ces derniers apparaissent plus résistants à la dessiccation que d'autres genres communs tels que le pin, l'épicéa ou le sapin, et peuvent survivre à des altitudes et dans des sites plus arides que certaines plantes moins bien adaptées.
Cette étude, menée sur une zone très localisée aux conditions climatiques et stationnelles assez marginales par rapport à l'aire d'extension méditerranéenne de l'espèce, révèle donc le déterminisme estival de la croissance radiale du genévrier thurifère et les potentialités dendrochronologiques de cette cupréssacée, pour la première fois abordée sous un aspect dendroécologique. Une étude dans toute son aire de répartition européenne mériterait d'être conduite, afin de mieux appréhender l'écologie de la sous-espèce européenne de ce genévrier.

L'étude dendroécologique, menée parallèlement sur les thuriféraies marocaines, devrait aussi nous apporter les premières données sur son comportement en milieu de haute montagne méditerranéenne et témoigner plus largement des potentialités de ce genévrier.

Remerciements : Ce travail a reçu le soutien financier de la communauté européenne (fonds européen PDZR) par l'intermédiaire de l'OGE (Office de génie écologique), que nous tenons à remercier ici.

Nous adressons également nos remerciements à Météo-France, qui a fourni les données météorologiques nécessaires à cette étude.

\section{Références}

[1] Aussenac G., Valette J.C., Comportement hydrique estival de Cedrus atlantica Manetti, Quercus ilex L. et Quercus pubescens Willd et de divers pins dans le Mont Ventoux, Ann. Sci. For. 39 (1) (1982) 41-62.

[2] Bagnouls F., Gaussen H., Saison sèche et indice xérothermique, Bulletin de la société d'histoire naturelle de Toulouse, Tome 88 (1953) 193-239.

[3] Bertaudière V., Montès N., Cartographie, dynamique et aperçu floristique du versant sud de la station à Juniperus thurifera de la montagne de Rié (Haute-Garonne, France), Mémoire de maîtrise, Univ. P. Sabatier, Toulouse, 1993, 55p.

[4] Bertaudière V., Montès N., Gauquelin T., Approche dendroécologique du Genévrier thurifère : exemple de la Montagne de Rié (Pyrénées, France) - Dynamique du peuplement et sensibilité au climat. ONF-Dossiers forestiers XX (sous presse) (1998)

[5] Ducrey M., Réactions à la sécheresse de quelques espèces méditerranéennes, Rev. For. Fr. XL 5 (1988) 361-370.

[6] Ducrey M., Adaptation du cèdre de l'Atlas (Cedrus atlantica Manetti) au climat méditerranéen : aspects écophysiologiques de sa réaction à la sécheresse, Ann. Rech. For. Maroc 27 (1) (1994) 140-153.

[7] Dupias G., La Montagne de Rié, Le Monde des Plantes 329 (1960) 3-5.

[8] Esper J., Bosshard A., Schweingruber F.H., Winiger M., Tree-rings from the upper timberline in the Karakorum as climatic indicators for the last 1000 years, Dendrochronologia 13 (1995) 79-88. 
[9] Eckstein D., Bauch J., Beitrag zur Rationalisierung eines dendrochronologischen Verfahrens und zur Analyse seiner Aussagesicherheit, Fortswiss. Centralbl. 88 (1969) 230-250.

[10] Fisher M., Is it possible to construct a tree-ring chronology for Juniperus excelsa (Bieb.) subsp. polvcarpos (K. Koch), Takhtajan from the northern mountains of Oman?, Dendrochronologia 12 (1994) 119-127.

[11] Fritts H.C., Tree Rings and Climate, Academic Press, London, 1976.

[12] Fromard F., Gauquelin T., Thuriferous juniper stands in Morocco: research and conservation for an endangered environment and species, Unasylva 44 (1) (1993) 52-58.

[13] Gauquelin Th., Lebreton Ph., Systématique de Juniperus thurifera $L$. : le cas de la population pyrénéenne de la Montagne de Rié (Haute-Garonne, France), J. Bot. Soc. Bot. Fr. 5 (1998) 105-109.

[14] Gauquelin Th., Bertaudière V., Montès N., Badri W., Asmodé J.F., Endangered stands of thuriferous juniper in the western Mediterranean basin: ecological status, conservation and management, Biodivers. Conserv. (Sous presse) (1999).

[15] Grissino-Mayer H.D., An updated list of species used in tree-ring research, Tree-Ring Bull. 53 (1993) 17-43.

[16] Guerby L., Une nouvelle station de Genévrier thurifère (Juniperus thurifera L) dans les Pyrénées : le Quié de Lujal (Ussal les Bains, Ariège), Le monde des plantes 447 (1993) $26-27$

[17] Guibal F., Dendroclimatologie du cèdre de l'Allas (Cedrus atlantica Manetti) dans le Sud-Est de la France, Ecol. Medit. XI (4) (1985) 87-103.

[18] Guiot J., The Boostrapped response function, Tree-ring Bull. 51 (1991) 39-41.

[19] Guiot J., Gœury C., PPPBase, a software for statistical analysis of paleoecological and paleoclimatological data, Dendrochronologia 14 (1996) 295-300.

[20] Guiot J., Berger A.L., Munaut AV, Till Cl., Some new mathematical procedures in dendroclimatology, with examples from Switzerland and Morocco, Tree-Ring Bull. 42 (1982) $33-48$

[21] Herman F.R., Growth and phenological observations of Arizona junipers, Ecology 37 (1) (1956) 193-194.

[22] Holmes R.L., Computer-assisted quality control in treering dating and measurement, Tree-Ring Bull. 43 (1983) 69-78.

[23] Kaennel M., Schweingruber F. (Compilers), Multilingual Glossary of Dendrochronology. Terms and Definitions in English, German, French, Spanish, Italian, Portuguese and Russian, Birmensdorf Swiss Federal Institute for Forest, Snow and Landscape Research, Bern, Paul Haupt, Stuttgart, Publishers, 1995.

[24] Larson P.R., The indirect effect of drought on tracheid diameter in Red Pine, Forest Sci. 9 (1) (1963) 52-62.

[25] Lathuillière L., Le Genévrier thurifère (Juniperus thurifera L). Monographie, Etude de la thuriféraie de St-Crépin. Le Genévrier thurifère dans le Sud-Est de la France. Mémoire de fin d'études, Engref, 1994, 80 p.
[26] Liphschitz N., Waisel Y., Lev-Yadun S., Dendrochronological investigations in Iran, Tree-Ring Bull. 39 (1979) 39-45.

[27] Messaoudène M., Tessier L., Relations cerne-climat dans des peuplements de Quercus afares Willd et Quercus canariensis Pomel en Algérie, Ann. Sci. For. 54 (1997), $347-358$

[28] Mölleken H., Eckstein D., Venne H., Scholz F., Growth of spruce (Picea abies L. Karst.) as controlled by genotype and cnvironment, Dendrochronologia 12 (1994) 23-32.

[29] Mon-Lin Kuo E.A., Mc Ginnes J.R., Variation of anatomical structure of false rings in castern redcedar, Woodscience 5 (3) (1973) 205-210.

[30] Munaut A.V., Recherches dendrochronologiques sur Pinus sylvestris. I. Étude de 45 pins sylvestres récents originaires de Belgique, Agricultura 14 (2) (1966) 193-232.

[31] Nefaoui M., Dendroécologie, productivité et dynamique de la croissance radiale du pin maritime naturel au Maroc, Tome 1 Dendroćcologie, thèse, univ de droit, d'économie et des sciences d'Aix-Marseille, 1996, 146 p.

[32] Petit A., Étude de Juniperus thurifera L. en Corse, mémoire de maîtrise de sciences et techniques, université de Corse, 1995, pp. 26-29.

[33] Safar W., Contribution à l'étude denroécologique du pin d'Alep (Pinus halepensis Mill) dans une région semi-aride d'Algéric : l'Atlas saharien (Ouled Nail - Aurès - Hodna), thèse, univ de droit, d'economie et des sciences d'Aix-Marseille, $1994,215 \mathrm{p}$.

[34] Santini A., Bottacci A., Gellini R., Preliminary dendroecological survey on pedonculate oak (Quercus robur L.) stands in Tuscany (Italy), Ann. Sci. For. 51 (1994) 1-10.

[35] Savoie JM., Les types de station forestière des Pyrénées centrales, ONF, 1995, pp. 11-78, 123-127.

[36] Schweingruber F.H., Tree Rings. Basics and Applications of Dendrochronology, Reidel Publishing Company, Holland, 1988.

[37] Schweingruber F.H., Eckstein D., Serre-Bachet F, Bräker O.U. Identification, presentation and interpretation of event years and pointer years in dendrochronology, Dendrochronologia 8 (1990) 9-38.

[38] Schweingruber F.H., Wehrli U., Aellen-Rumo K., Aellen M., Weiserjahre als Zeiger extremer Standortseinflüsse, Schweiz. Z. Forstwes. 142 (1) (1991) 33-52.

[39] Serre-Bachet F., Analyse dendroclimatologique comparée de quatre espèces de pins et du chêne pubescent dans la région de la Gardiole près Rians (Var, France), Ecol. Medit. VIII (3) (1982) 167-183.

[40] Serre-Bachet F., Tessier L., Response Function Analysis for Ecological Study, in : Cook E.R., Kairiukstis L.A. (éd.), Methods of Dendrochronology. Applications in the Environmental Sciences, Kluwer Academic Publishers, Dordrecht, 1990, pp. 247-258.

[41] Stoke M., Smiley T.L., An Introduction to Tree-Ring Dating, The University of Chicago Press, Chicago, London, 1968. 
[42] Tessier L., Dendroclimatologie et Ecologie de Pinus sylvestris L. et Quercus pubescens Willd dans le Sud-Est de la France, thèse de doctorat d'État, université Aix-Marseille III, $1984,275 \mathrm{p}$.

[43] Tessier L., Approche dendroclimatologique de l'écologie de Pinus sylvestris L. et Quercus pubescens Willd. Dans le Sud-Est de la France, Oecol. Plant. (21) (1986) 339-355.

[44] Tessier L., Serre-Bachet F., Chênes caducifoliés en région méditerranéenne : relations cerne-climat, Ecol. Medit. XVI (1990), 209-221.

[45] Tessier L., Pons A., Serre-Bachet F., Analyse dendroclimatologique comparée de quelques populations de chêne pubescent (Quercus pubescens Willd.) de la Drôme et du Var, Ecol. Medit. VIII (4) (1982) 117-130.
[46] Waisel Y., Liphschitz N., Dendrochronological studies in Israël. II: Juniperus phonicea of north and central Sinaï, La Yaaran 18 (1968), 67-69.

[47] Yamaguchi D.K., A simple method for crossdating increment cores from living trees, Can. J. Forest Res. 21(1991) 414-416.

[48] Zhang S.H., Contribution à l'étude de la croissance en diamètre du chêne vert (Quercus ilex L.) en relation avec le climat, thèse, univ des sciences et techniques du Languedoc, Montpellier, 1987, $183 \mathrm{p}$.

[49] Zhang S.H., Romane F., Variations de la croissance radiale de Quercus ilex 